\title{
Development of Learning and Memory in Aplysia. III. Central Neuronal Correlates
}

\author{
Thomas G. Nolen, Emilie A. Marcus, and Thomas J. Carew \\ Departments of Psychology and Biology, Yale University, New Haven, Connecticut 06520
}

The defensive withdrawal reflex of the mantle organs of Aplysia californica has 2 major components, siphon withdrawal and gill withdrawal. In the previous paper of this series (Rankin and Carew, 1987), the development of 2 forms of nonassociative learning, habituation and dishabituation, was examined in the siphon withdrawal component of the reflex. In the present study we examined these same forms of learning in the gill withdrawal component of the reflex. The purpose of these experiments was 2-fold: (1) to examine the development of learning in the other major component of the reflex; and (2) to establish preparations in which it is possible to carry out a cellular analysis of the development of learning in the CNS.

We first established that the gill withdrawal reflex in intact animals exhibited significant habituation in response to repeated tactile stimulation of the siphon and significant dishabituation in response to tail shock. We next determined the contribution of the CNS to the gill withdrawal reflex by surgically removing the abdominal ganglion from intact animals. Using the same stimulus intensity $(4 \mathrm{mg})$ that produced habituation in the previous experiments, we found that the CNS accounted for approximately $95 \%$ of the reflex.

Finally, we developed 2 preparations that allowed us to relate behavioral observations of learning directly to neural plasticity exhibited in the CNS. In a semi-intact preparation gill withdrawal was behaviorally measured as in the intact animal, but tactile stimulation of the siphon (to produce habituation) and shock to the tail (to produce dishabituation) were replaced by electrical stimulation of the siphon nerve and left connective, respectively. Stimulation parameters were matched to produce behavioral responses comparable with those in the intact animal. In an isolated CNS preparation the same nerve stimuli were used as in the semiintact preparation, but the response measure used was the evoked neural discharge recorded in an efferent nerve innervating the gill. Both preparations exhibited response decrement and facilitation that was quantitatlvely as well as qualitatively similar to that observed in intact animals, in-

\footnotetext{
Received Apr. 1, 1986; revised June 30, 1986; accepted July 1, 1986.

We wish to thank Deda Gillespie for her great help with the data analysis and our colleagues Catharine Rankin and Mark Stopfer for their helpful comments throughout the project. We also thank the Howard Hughes Medical Institute and Tom Capo for generously supplying juvenile Aplysia. This work was supported by NIH CDA Award 7-K02-MH00081-09, NIH BRSG Grant 507-RR-075015, and NSF Grant BNS 8311300 (T.J.C.) and NIH NRSA 5-F32-NS07480 (T.G.N.).

Correspondence should be addressed to Thomas J. Carew, Department of Psychology, Yale University, P.O. Box 11A, Yale Station, New Haven, CT 06520.

Copyright (C) 1987 Society for Neuroscience 0270-6474/87/010144-10\$02.00/0
}

dicating that 2 simple forms of learning exhibited by the gill withdrawal reflex in juvenile Aplysia can be localized to neural circuits within the abdominal ganglion.

The analysis of learning from a developmental perspective has provided a valuable approach towards establishing important relationships between different forms of learning and memory in a number of mammalian systems (see, e.g., Campbell and Spear, 1972; Camphell and Coulter, 1976; Amsel and Stanton, 1980). However, because of the complexity of these systems it has not yet been possible to carry this developmental approach to a cellular level where a direct comparison of different forms of learning can be made in mechanistic terms. Such an analysis requires a preparation that not only exhibits different forms of learning and memory early in development but also has a nervous system that is amenable to cellular investigation at the same early developmental stages. We have found that the marine mollusc Aplysia californica provides an excellent preparation in which to combine developmental and cellular approaches in the analysis of learning and memory.

We have focused our experimental efforts on the gill and siphon withdrawal reflex of Aplysia because it offers several advantages for a developmental study of lcarning. The reflex emerges early in juvenile ontogeny and can be readily elicited and quantified as soon as it appears (Rankin et al., 1987). Moreover, different forms of nonassociative learning and memory emerge sequentially and systematically in the siphon withdrawal component of this reflex (Rankin and Carew, 1987).

In this paper we examine the gill withdrawal component of the defensive withdrawal reflex in juvenile Aplysia. As a first step towards a cellular analysis, we have developed several preparations in which it is possible to relate learning observed at a behavioral level directly to neuronal plasticity observed in the central nervous system (CNS). Specifically, we first established that the gill withdrawal reflex exhibits 2 simple forms of learning (habituation and dishabituation) comparable with those observed in the siphon withdrawal component of the reflex at the same developmental stage (Rankin and Carew, 1987). Next, we assessed the central contribution to the reflex by surgical removal of the abdominal ganglion from intact animals. Using the same tactile stimulation to the siphon that produced habituation in the intact animal, we found that the reflex was mediated virtually entirely ( $95 \%)$ by the CNS. Finally, we developed 2 preparations that have allowed us to specify that habituation and dishabituation in juvenile Aplysia are centrally mediated and can be localized to circuits within the abdominal ganglion. A preliminary account of some of these results has heen reported in abstract form (Nolen et al., 1985). 


\section{Materials and Methods}

Experimental animals. Thirty-two juvenile $A$. californica were used in this study. All animals were laboratory-cultured stage 11 juveniles obtained from the Howard Hughes Mariculture Facility, Woods Hole, MA. Staging was assessed on the basis of the external morphological criteria described by Kriegstein (1977a; Kriegstein et al., 1974; see also Rankin et al., 1987). Specifically, stage 11 animals range in length from 2 to $5 \mathrm{~mm}(400 \mu \mathrm{g}$ to $1.5 \mathrm{mg})$, have small immature rhinophore buds, and lack a genital groove. The animals were maintained at $15^{\circ} \mathrm{C}$ and were housed in small groups $(n=5-10)$ in $50 \mathrm{ml}$ centrifuge tubes containing Woods Hole seawater and the red alga Gracillaria.

\section{Intact behavior}

Behavioral procedures. One day before an experiment, animals were restrained with micropins inserted through their parapodia in a Sylgardcoated petri dish and their shells were removed with fine forceps. Removal of the shell allowed the mantel shelf to retract and maximized exposure of the gill. On the day of the experiment, animals were placed on an angled Sylgard ramp (approx. $\left.30^{\circ}\right)$ in a petri dish $(60 \times 15 \mathrm{~mm}$ ) containing freshly aerated artificial seawater (ASW; Instant Ocean) and were again restrained with micropins through the parapodia. The orientation of the animal on the ramp and the nature of the restraint (slightly rotating the animal) optimized visualization of the gill and allowed its responses to be scored accurately.

Behavioral observations were videotaped under dark-field illumination using a Zeiss SV8 stereomicroscope fitted via a trinocular head to a camera (JVC S-62U), which was in turn connected to a videocassette recorder (Panasonic NV-8950) and a color video monitor (Sony) (for details, see Rankin et al., 1987). The stimulus used to evoke a reflex gill contraction consisted of a short, quantifiable water jet $(4 \mathrm{mg}, 50$ msec) delivered to the siphon via a micropipette connected to a Picospritzer II (General Valve Corp.). (In some cases, a shorter, $5 \mathrm{msec}$, pulse was used-see Results.) The pipette was positioned with a micromanipulator so that it was aimed directly at the siphon. Stimulus intensity was adjusted for each animal by varying the distance of the pipette from the siphon so that the water jet produced a moderate gill contraction (see Results). Dishabituating stimuli were brief electrical pulses (a $2.5 \mathrm{sec}$ train of $20 \mathrm{msec}$ pulses at $10 \mathrm{pulses} / \mathrm{sec}$ ) delivered to the tail via a suction electrode using a Grass S88 stimulator. Efficacy of the tail shock was assessed for each animal by delivering a single shock ( $20 \mathrm{msec}$ pulse) to the tail prior to the habituation run and assessing reflex gill and body contractions and/or inking. The animals were then rested for $15 \mathrm{~min}$ before the start of the habituation trials. Habituation was produced by delivering 30 water jet stimuli to the siphon at a $10 \mathrm{sec}$ interstimulus intcrval (ISI). Dishabituation was produced by delivering a single train of stimuli to the tail, followed by 15 more tactile stimuli at the same $10 \mathrm{sec}$ ISI.

Gill contraction amplitude was measured using a point-to-point measure. For each animal, the experimenter chose 2 clearly visible points on the gill, one at the lateral margin (which would move inward during a contraction) and another at a distant fixed point (which would not move during a contraction). Then, using stop-frame and slow-motion playback, the distance between the 2 points was measured just prior to delivery of each stimulus (i.e., when the gill was relaxed) and at the peak of each contraction. The amplitude of each response was expressed as the percentage difference between the relaxed and contracted distances.

Assessment of the central contribution to the reflex. An intensity of the water-jet stimulus to the siphon was selected (4 mg; see Rankin et al., 1987) that produced a moderate gill contraction comparable both with those used to study habituation (as described above) and with the stimulus intensity used to study habituation of siphon withdrawal (Rankin et al., 1987). The distance between the micropipette and the siphon was then recorded so that, for each animal, the postsurgery stimulus intensity could be reliably matched to the presurgery intensity. Fifteen minutes after a stimulus intensity had been determined, 5 stimuli were delivered to the siphon at a $3 \mathrm{~min}$ ISI. The animals were then anesthetized by placing them in $100 \%$ isotonic $\mathrm{MgCl}_{2}(367 \mathrm{mM})$ for $15 \mathrm{~min}$. The animals were then pinned out ventral side up in a Sylgard-coated petri dish containing ASW. A midline incision was made along the length of the foot, and the gut and esophagus were removed. In deganglionated animals the abdominal ganglion was then excised by cutting the pleuroabdominal connectives and the peripheral nerves (branchial, genital-pericardial, and siphon) with micropipettes. In sham-operated control animals, the abdominal ganglion was identified and the integrity of its peripheral nerves and connectives was verified. The animals were then unpinned (without sealing the midline incision) and allowed to recover from the anesthesia in normal ASW at room temperature for $1 \mathrm{hr}$, at which point both groups showed normal head and body contractions and buccal mass movements indicative of recovery from anesthesia. The animals were then repinned in the test dish on the angled Sylgard ramp, rested for $15 \mathrm{~min}$, and presented with 5 more siphon stimuli at a $3 \mathrm{~min}$ ISI. Double-blind procedures were used to collect and analyze the data: The experimenter setting up the animals and delivering the pre- and postsurgery stimuli did not know the experimental condition of the animal. The responses were then scored and digitized (see Rankin and Carew, 1987; Rankin et al., 1987) by a second experimenter who also did not know the condition of the animals.

Five test stimuli were used to obtain a reliable estimate of reflex amplitude. To control for any effects of repeated stimulation, the data for both deganglionated and sham-operated animals were normalized by expressing the response to each of the 5 test stimuli in the postsurgery test as a percentage of the corresponding stimulus in the presurgery test. Each animal then contributed a single score (net percentage change), which was the average of the percentage change exhibited to the 5 stimuli.

\section{Semi-intact preparation}

Animals were anesthetized, pinned out, and dissected as described above. The esophagus and gut were removed to reveal the abdominal ganglion. The pleuroabdominal connectives were cut close to the pleural ganglia. The siphon nerve was traced from the ganglion to the place where it innervates the periphery and was then cut at this most distal site to provide the longest length of nerve possible (approx. $0.2 \mathrm{~mm}$ ). This left the abdominal ganglion connected to the periphery solely by the branchial nerve. The floor of the mantle cavity was then cut to expose the gill. The gill and abdominal ganglion were then removed.

The end of the siphon nerve was drawn into the tip of a glass microelectrode (tip diameter, 20-50 $\mu \mathrm{m}$ I.D.) filled with ASW and connected to a stimulator. Gill contractions were evoked by stimulating the siphon nerve with a single pulse $(5 \mathrm{msec}, 1-15 \mathrm{~V})$. A second suction electrode was placed on the left connective, which carries the input from the tail into the abdominal ganglion. This electrode was used to deliver the dishabituating stimulus (a $2.5 \mathrm{sec}$ train of $5 \mathrm{msec}$ pulses at 10 pulses/ $\mathrm{sec}$ ). The intensity of the siphon nerve stimulus was adjusted to produce a moderate gill contraction comparable with those produced in the intact animal in response to tactile stimulation of the siphon. To assess the efficacy of the facilitating stimulus, the stimulus intensity to the left connective was incrcascd until a single shock produced a gill contraction. The preparation was then rested for $15 \mathrm{~min}$. To produce habituation, 15 stimuli were delivered to the siphon nerve at a $10 \mathrm{sec}$ ISI, followed by a single train of stimuli to the connective and then by 10 more stimuli to the siphon nerve at the same $10 \mathrm{sec}$ ISI. The evoked gill responses were scored as described above.

\section{Isolated CNS preparation}

The procedure used to excise the abdominal ganglion was identical to that described above except that in this instance the branchial nerve was also cut at the distal end near its insertion into the gill. Again, suction electrodes on the siphon nerve and left connective were used to deliver decrementing and facilitating stimuli, respectively. The response measured in these experiments was an evoked discharge in the branchial nerve, which carries the efferent output to the gill. The discharge was recorded via a third suction electrode connected to a Grass P511 extracellular amplifier $(50,000 \times$ gain $)$. All data were tape-recorded on a Hewlett-Packard 3968A FM tape recorder. Siphon nerve stimulus intensity (in the range of intensities used in the semi-intact preparation described above) was adjusted until it produced a clear evoked efferent response of approximately $1 \mathrm{sec}$ duration. To ensure an effective facilitating stimulus, the connective stimulus intensity was increased until it produced a small efferent response in the branchial nerve. The preparation was then rested $15 \mathrm{~min}$. To produce response decrement, 15 stimuli were delivered to the siphon nerve (10 sec ISI). A single train of facilitating stimuli (a $2.5 \mathrm{sec}$ train of $5 \mathrm{msec}$ pulses at $10 \mathrm{pulses} / \mathrm{sec}$ ) was then delivered to the connective, followed by 10 more siphon nerve stimuli at the same $10 \mathrm{sec}$ ISI. 


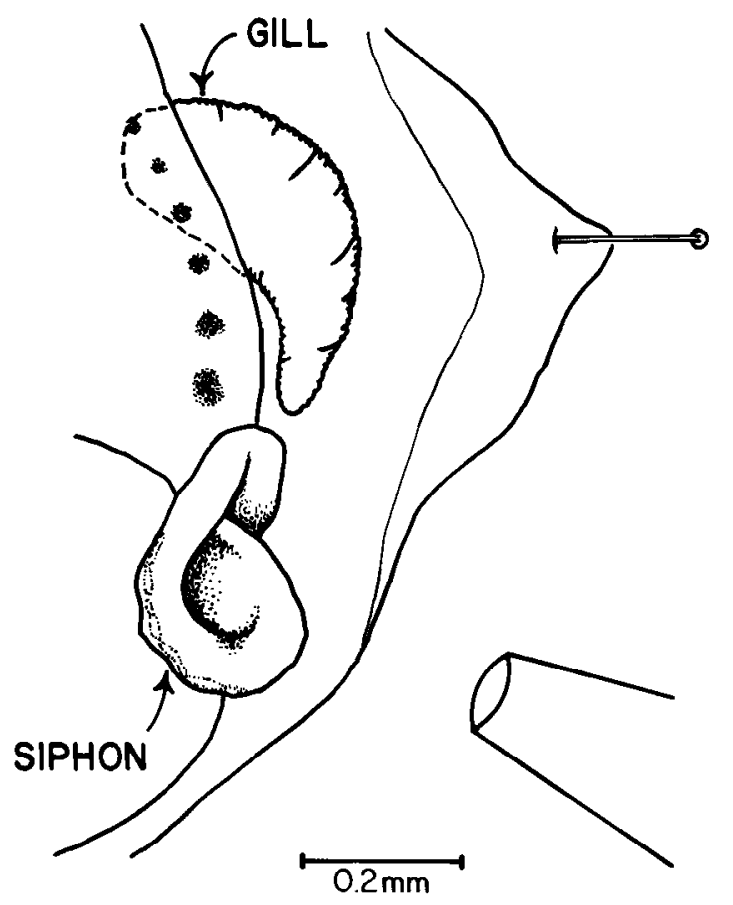

Figure 1. Preparation for studying gill withdrawal in stage 11 juveniles. Animals were restrained with pins through their parapodia. The gill withdrawal reflex was triggered with a water jet stimulus from a micropipette (lower right) directed at the siphon. (See text for details.)

For scoring, the tape-recorded efferent discharges were played back into a Gould pen recorder at $1 / 8$ speed. At this speed the frequency response of the pen recorder was sufficient to allow resolution of single extracellular action potentials. The evoked discharges were quantified using a digitizing tablet to generate a poststimulus time (PST) histogram. For each experiment, a baseline was determined from the prestimulus (background) records. Spikes whose amplitude exceeded this baseline were considered part of the evoked discharge, and their latency relative to the delivery of the stimulus was measured and entered into the ongoing real-time record. This yielded a PST histogram of the number of spikes (expressed in $250 \mathrm{msec}$ bins) that occurred before and after the stimulus. The $250 \mathrm{msec}$ bins were chosen because they permitted sampling of a relatively long response period while retaining resolution of the temporal structure of the evoked burst. In the analysis of decrement and facilitation, the total number of spikes occurring in the first $750 \mathrm{msec}$ following a stimulus was recorded for each response.

\section{Statistical analysis}

All habituation data were normalized by expressing each response as a percentage of the initial response. Magnitude of habituation was determined by computing, for each animal, the difference between the last response and the initial response of a habituation run. The magnitude of dishabituation was determined by computing the difference between the last response in the habituation run and the first response following tail shock. Except where noted, within-group and between-group comparisons were made with $t$ tests for correlated or independent means, respectively (Winer, 1962). In 1 experiment (habituation in the semiintact preparation), all 5 animals yielded a normalized initial score of $100 \%$ and a final habituation score of $0.0 \%$; thus, a parametric analysis on these data was inapplicable. In this case the raw (nonnormalized) scores for cach animal wcre used for the within-group analysis by a nonparametric Wilcoxon signed-ranks test for paired comparisons. Unless otherwise indicated, all probability values are 2-tailed. Data are presented in terms of means \pm SEM.

\section{Results}

Gill withdrawal in intact animals

The gill withdrawal reflex of juvenile stage 11 animals was examined in restrained animals by stimulating the siphon with a

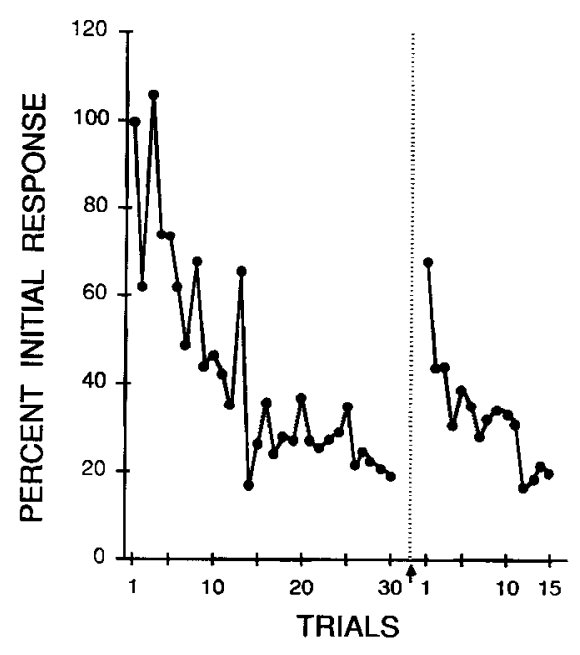

Figure 2. Habituation and dishabituation of the gill withdrawal reflex in intact animals. Thirty water jet stimuli presented at a $10 \mathrm{sec}$ ISI produced significant habituation $(n=6)$. Tail shock (arrow and dotted line) produced significant dishabituation. Data in this and other summary figures are expressed as the mean percentage of the initial response.

brief water jet from a micropipette and recording the response of the gill (Fig. 1; see Materials and Methods). A stimulus intensity of $4 \mathrm{mg}$ was chosen to produce gill reflex contractions of moderate amplitude, about a $15 \%$ change in the gill's linear dimension.

\section{Habituation and dishabituation}

Previous studies showed that the siphon withdrawal component of the defensive withdrawal reflex in stage 11 animals exhibited significant habituation to stimuli presented at a $10 \mathrm{sec}$ ISI and significant dishabituation in response to tail shock (Rankin and Carew, 1987). In our first experiment, we examined habituation and dishabituation of the gill withdrawal component of the reflex in the same developmental stage. We found that the gill withdrawal reflex also showed significant habituation to a 10 sec ISI in stage 11 animals $(n=6)$. The results are shown in Figure 2. Gill response amplitude is plotted across trials as a percentage of the initial response. Repeated stimulation at a 10 sec ISI produced a significant decrement in response amplitude $(t,=32.35, p<0.001)$, with the habituated response amplitude roughly asymptotic at about $25 \%$ of the initial response by the 20 th stimulus. Dishabituation in the gill withdrawal component of the reflex was also apparent at this stage of development. Tail shock produced significant facilitation of the habituated reflex gill contraction ( $t_{5}=2.04, p<0.05,1$-tailed) (Fig. 2). Tail shock evoked both gill and siphon contractions in all preparations, as well as inking in most. The dishabituated response rehabituated with successive stimulation at the same $10 \mathrm{sec}$ ISI (Fig. 2).

\section{Contribution of the CNS to the gill withdrawal reflex}

An important first step in developing preparations that allow a cellular analysis of learning in different stages of ontogeny is to determine the relative contribution of the CNS to the gill withdrawal reflex in juvenile animals. To explore this question, we examined the effects of removing the abdominal ganglion in stage 11 animals.

Animals were randomly assigned to 1 of 2 groups: deganglionated $(n=7)$ or sham-operated $(n=7)$. Prior to surgery, all animals were given a series of 5 water jet stimuli at a long ( 3 


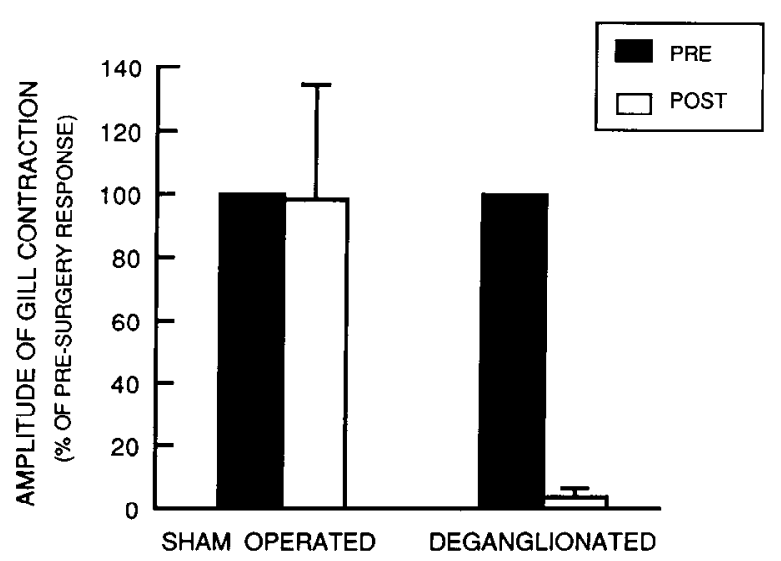

Figure 3. Contribution of the CNS to the gill withdrawal reflex. Data are expressed as the mean percentage change in response $( \pm$ SEM $)$ in the postsurgery test (white bars) compared with the presurgery test (black bars) (see Materials and Methods). Sham-operated controls $(n=7)$ showed no significant change in their reflex response, whereas deganglionated animals $(n=7)$ showed a profound reduction in reflex amplitude (to $5.1 \%$ of presurgery levels).

min) ISI to avoid habituation. The intensity of the stimulus (about $4 \mathrm{mg}$ ) was adjusted to produce responses of modest amplitude, comparable with those produced in the habituation experiment described above. There was no significant difference in response amplitude between the initial response exhibited in the habituation experiment $(\bar{X}=13.03 \pm 2.5, n=6)$ and the presurgery responses in the present experiment $(\bar{X}=11.02 \pm$ $1.14, n=14 ; t_{18}=0.85, p>0.5$ ).

The results of deganglionation on the evoked gill withdrawal response is shown for 7 pairs of animals in Figure 3. The data are expressed in terms of a score that reflects the net percentage change in response in the postsurgery test compared with the presurgery test (see Materials and Methods). Although there was considerable variability, the average response of the sham-operated controls following surgery was virtually unchanged $(\bar{X}=$ $98.1 \pm 35.8 \%$ ) compared with the presurgery response. In contrast, removal of the abdominal ganglion significantly and dramatically reduced the response to an average of only $5.1 \pm 2.6 \%$ of that before surgery $\left(t_{6}=36.44, p<0.001\right)$. Moreover, the response reduction exhibited by the deganglionated group was significantly greater than that exhibited by the sham-operated controls $\left(t_{12}=2.58, p<0.025\right)$. These results demonstrate that, with the stimulus intensity used in these experiments, the CNS in stage 11 juvenile animals accounts for approximately $95 \%$ of the gill withdrawal reflex.

\section{Habituation and dishabituation in semi-intact preparations}

Having established that the abdominal ganglion mediates the gill withdrawal reflex, we next wished to investigate whether the abdominal ganglion also mediated the 2 simple forms of learning, habituation and dishabituation, exhibited in stage 11 animals (Fig. 2). We examined this question in a surgically reduced (semi-intact) preparation consisting of the excised abdominal ganglion attached to the gill via the branchial nerve, as shown in Figure 4. At this stage of development, the ganglion is about $200 \mu \mathrm{m}$ in diameter and the gill about $800 \mu \mathrm{m}$ in length. Reflex contractions of the gill were elicited by delivering a brief shock to the siphon nerve with a suction electrode. The siphon nerve was used to elicit the reflex because it carries the afferent input from the siphon to the CNS.

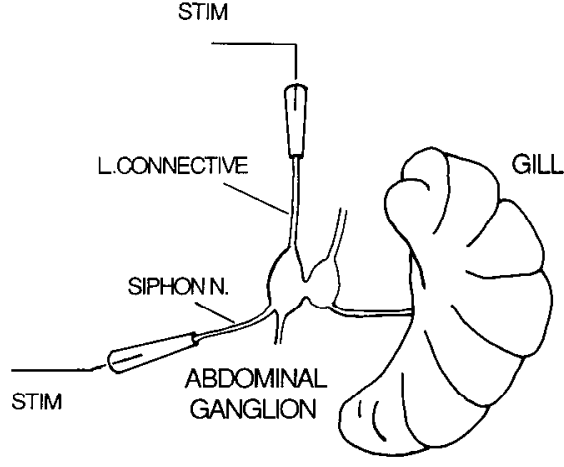

Figure 4. Semi-intact preparation. The abdominal ganglion was removed, leaving it attached to the gill by the branchial nerve. The reflex response was elicited and habituated by repeated electrical stimulation of the siphon nerve. Dishabituation was produced by stimulating the left connective, which carries the input from the tail to the abdominal ganglion (sce text).

To compare habituation and dishabituation in intact and semiintact preparations, it was important to functionally equate electrical stimulation of the siphon nerve in the semi-intact preparation with tactile stimulation of the siphon skin in the intact animal. We accomplished this by selecting a stimulus intensity to the siphon nerve that produced gill contractions comparable with those elicited in intact animals by water jet stimulation of the siphon. There was no significant difference in the amplitudes of evoked gill contractions in the intact $(\bar{X}=13.03 \pm 2.5, n=$ 6) and semi-intact preparations $\left(\bar{X}=8.90 \pm 1.37, n=6 ; t_{10}=\right.$ $1.45, p>0.10)$.

The stimulus intensity to the siphon nerve determined above was used to produce habituation. A single train of electrical shocks (a $2.5 \mathrm{sec}$ train of $5 \mathrm{msec}$ pulses at 10 pulses/sec) delivered to one of the pleuroabdominal connectives served as the dishabituating stimulus. The pleuroabdominal connectives were chosen because they are known to carry input from the tail to the abdominal ganglion in adults (Walters et al., 1983). Such a train of pulses to one of the connectives evoked a strong gill contraction, similar to that produced by tail shock in the intact animal. Since both siphon nerve and connective stimulation elicited gill withdrawal, these results show that both centrally

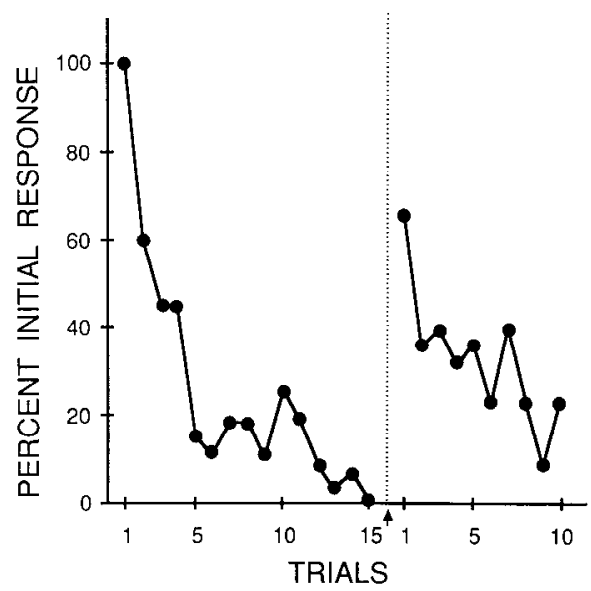

Figure 5. Habituation and dishabituation in the semi-intact preparation. Repeated stimulation of the siphon nerve (ISI $=10 \mathrm{sec}$ ) produced significant habituation. A single train of stimuli to the left connective (arrow and dotted line) produced significant dishabituation $(n=5$ ). 


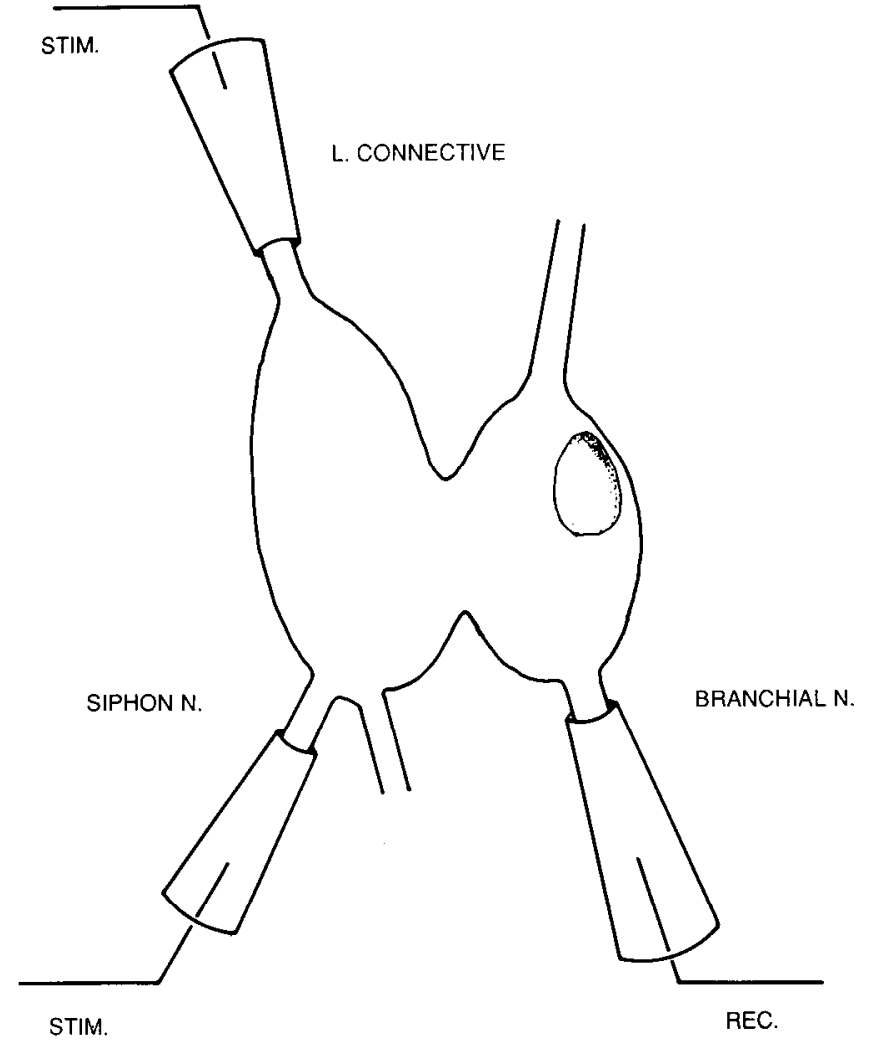

Figure 6. Isolated abdominal ganglion preparation. Reflex output was recorded as the discharge of efferent neurons in the branchial nerve. Siphon nerve stimulation served to trigger the reflex discharge and connective stimulation to provide facilitating input (as in the semi-intact preparation). For scale, the ganglion is approximately $200 \mu \mathrm{m}$ wide.

projecting siphon afferents and facilitatory inputs make functional connections with gill motor neurons as early as stage 11 juveniles.

Habituation in the semi-intact preparation $(n=5)$ is shown in Figure 5. Repeated stimulation of the siphon nerve at a 10 sec ISI produced significant habituation within 15 stimuli $(p<$ 0.03 ). Furthermore, a single train of stimuli to the connective produced significant dishabituation of the evoked response $(p<$ $0.03)$, similar to that produced by tail shock in the intact animal (compare Fig. 5 with Fig. 2). As in the intact animal, the dishabituated response in the semi-intact preparation subsequently rehabituated with continued stimulation at the same 10 sec ISI. Our data thus show that simple forms of learning in the semiintact preparation are qualitatively similar to those observed in intact animals at this stage of development. In both cases, repeated afferent stimulation (either by tactile stimulation of the siphon or by electrical stimulation of sensory afferents in the siphon nerve) produced habituation, while activation of facilitatory pathways (by either direct electrical stimulation of the tail or activation of tail input in the pleuroabdominal connectives) produced dishabituation.

\section{Response decrement and facilitation in the isolated abdominal ganglion}

The above experiments strongly suggest that, just as the gill withdrawal reflex is centrally mediated in stage 11 juveniles, habituation and dishabituation are also centrally mediated. However, since the abdominal ganglion was still attached to the gill (via the branchial nerve) in the semi-intact preparation, the possibility remained that the habituation and dishabituation might be mediated, at least in part, by peripheral processes in the gill itself. To explore this possibility, we examined response decrement and facilitation (reflecting central processes underlying habituation and dishabituation, respectively) in the isolated abdominal ganglion. Our procedures were identical to those used in the semi-intact preparation with the exception that, rather than recording gill contractions, we recorded the efferent discharge in the branchial nerve in response to siphon nerve stimulation (Fig. 6). Results from the semi-intact preparation permitted us to choose stimulation parameters for the afferent input (via the siphon nerve) and for the facilitating input (via

\section{DECREMENT}

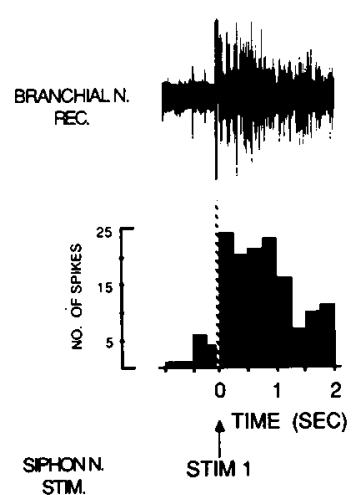

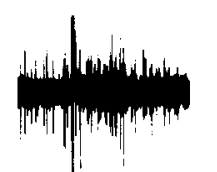

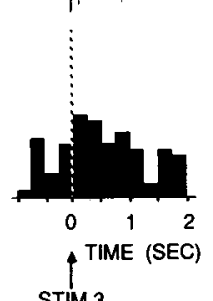

STIM 3
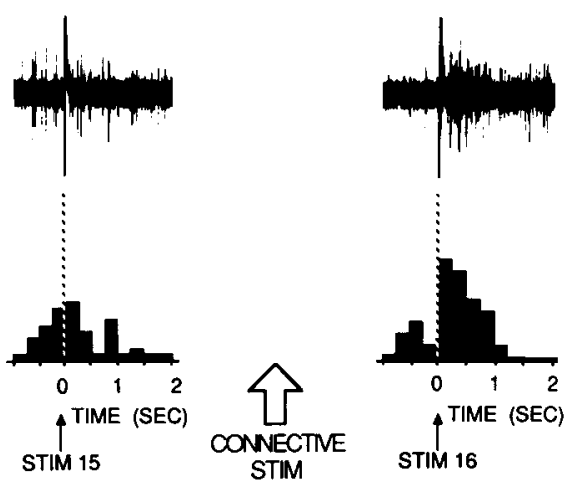

\section{FACILTATION}

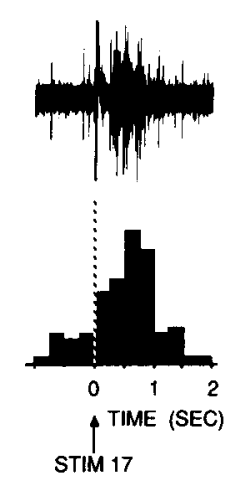

Figure 7. Decrement and facilitation in the isolated CNS. Top traces, Extracellular records of efferent (reflex) discharge recorded in the branchial nerve in response to siphon nerve stimulation. Bottom traces, PST histograms of efferent discharge. In each pair of traces, 1 sec of baseline is shown, followed by a $2 \mathrm{sec}$ record of the response to the siphon nerve stimulus (solid arrow and dotted line). At left, 15 siphon nerve stimuli (10 sec ISI) produced significant decrement (1st, 3rd, and 15th responses are shown). Following connective stimulation (open arrow), significant facilitation of the efferent response was observed (at right). Note the buildup of facilitation in the 16th-18th responses. 


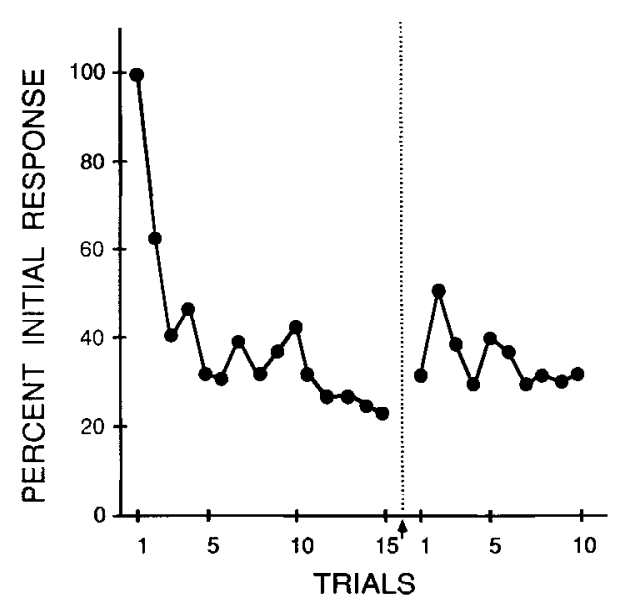

Figure 8. Summary of isolated CNS experiments. Repeated siphon nerve stimulation (ISI $=10 \mathrm{sec}$ ) produced significant decrement. Following connective stimulation (arrow and dotted line), significant facilitation was produced $(n=7)$.

the connectives) that were of known behavioral relevance. Thus, a brisk branchial nerve discharge was elicited by the same intensity and duration of electrical stimulus (1-15 V, $5 \mathrm{msec})$ to the siphon nerve (Fig. 7) that elicited a brisk gill withdrawal reflex in the semi-intact preparation. Latencies for the evoked nerve response were on the order of $20-50 \mathrm{msec}$; the response usually consisted of a burst of about 100 action potentials in the first $1000 \mathrm{msec}$ following the stimulus, which decreased to about 25 action potentials in the next $1000 \mathrm{msec}$.

Comparable to habituation of the reflex response, decrement of the branchial nerve discharge was produced by repeated stimulation of the siphon nerve (15 stimuli at a $10 \mathrm{sec}$ ISI). Comparable with dishabituation, facilitation of the efferent discharge was assessed by delivery of a single train of electrical stimuli (a $2.5 \mathrm{msec}$ train of $5 \mathrm{msec}$ pulses at 10 pulses/sec) to the connectives followed by 10 more siphon nerve stimuli at the same $10 \mathrm{sec}$ ISI. All stimulation parameters were identical to those used in the semi-intact preparation.

The extracellularly recorded branchial nerve response was quantified by means of PST histograms, in which the number of action potentials in the first $2 \mathrm{sec}$ following siphon stimulation was computed (see Materials and Methods). An example of a typical experiment is shown in Figure 7. In this example, the results for stimulus trials 1,3 , and 15 are shown on the left, while the first 3 trials following connective stimulation are shown on the right. At the top are the extracellularly recorded branchial nerve responses. These records were used to make the PST histograms shown at the bottom. Each record consists of $1 \mathrm{sec}$ of baseline activity followed by $2 \mathrm{sec}$ of response, with the vertical marker indicating the time of presentation of the siphon nerve stimulus. Note the clear decrement of response following repeated stimulation and the clear facilitation of the response following connective stimulation. This example also illustrates a common feature of dishabituation in adults, the buildup in response facilitation during the first few stimuli following connective stimulation.

A summary of our experiments $(n=7)$ examining decrement and facilitation of the evoked branchial nerve response is shown in Figure 8. Evoked responses were quantified as the number of action potentials occurring in the first $750 \mathrm{msec}$ following

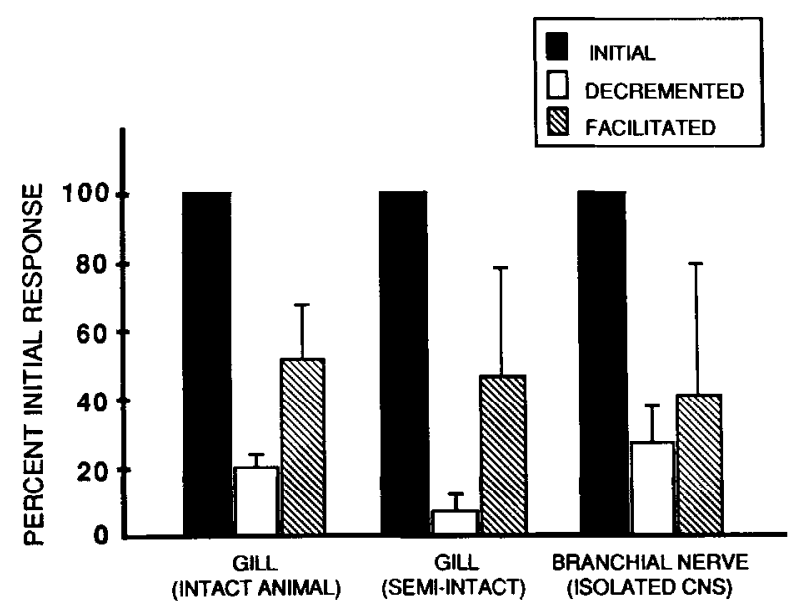

Figure 9. Comparison of decrement and facilitation in intact, semiintact, and isolated CNS preparations. In each case the initial response (black bar) is taken to be $100 \%$. The decremented response (white bar) is the mean of the last 3 responses of the habituation trials. The facilitated response (hatched bar) is the mean of the first 3 trials following tail shock (intact animal) or connective stimulation (semi-intact and isolated CNS). Qualitatively similar decrement and facilitation are observed in all 3 preparations.

siphon nerve stimulation. All responses were then expressed as a percentage of the initial response. As in the semi-intact preparation (Fig. 5), repeated stimulation of the siphon nerve at a $10 \sec$ ISI for 15 trials produced a significant decrement of the evoked branchi nerve efferent discharge $\left(t_{7}=10.1, p<0.001\right)$. Moreover, stimulation of the connectives produced significant facilitation of the decremented evoked response $\left(t_{6}=2.17, p<\right.$ $0.05,1$-tailed). The facilitated response was initially proportionately lower than that seen in intact animals $(30 \%$ of initial response compared with $50 \%$ ) but reached similar levels by the second or third stimulus (Fig. 8).

Our experiments with successively reduced preparations allow a direct comparison of simple forms of learning in the gill withdrawal reflex and their central neural correlates in the abdominal ganglion. The qualitative similarity of the plasticity in these preparations is shown in Figure 9, which illustrates (1) the results from intact animals, in which gill contractions were evoked by tactile stimulation of the siphon; (2) the results from the semi-intact preparation, in which gill contractions were evoked by siphon nerve stimulation; and (3) the results from the isolated abdominal ganglion, in which the efferent output to the gill in the branchial nerve was recorded in response to siphon nerve stimulation. For each of these preparations, the data have been normalized as a percentage of the initial response. In each case, a significant and qualitatively similar response decrement was produced by repeated afferent input, and significant response facilitation was produced by a strong stimulus either to the tail or to central pathways carrying tail input.

An important feature of these experiments is that stimuli were first established as behaviorally relevant in intact animals and then successively translated into progressively more reduced preparations. The conclusion that the stimuli were functionally equivalent is supported by the similarity of the response plasticity observed in each preparation. Thus, taken collectively, the results show that, at this stage of development, components of the underlying mechanisms of habituation and dishabituation are centrally mediated and localized to the abdominal ganglion. 


\section{Discussion}

Learning and memory in the gill withdrawal reflex of juvenile Aplysia

The defensive withdrawal reflex of Aplysia has 2 principal components, siphon withdrawal and gill withdrawal. In the first paper of this series, Rankin and Carew (1986) examined the siphon withdrawal component of the reflex in juvenile Aplysia and found that 2 simple forms of nonassociative learning, habituation and dishabituation, emerge sequentially during ontogeny, habituation (in stage 9) preceding dishabituation (in stage 10) by about 1 week. In the present study we examined these same forms of learning in the gill withdrawal component of the reflex in juvenile Aplysia. The purpose of these experiments was 2-fold: first, we wished to examine the development of learning in the other major component of the defensive withdrawal reflex; and second, we wished to establish preparations in which it is possible to carry out cellular studies of the development of learning in the CNS.

\section{Habituation and dishabituation of gill withdrawal}

A weak tactile water jet stimulus to the siphon of stage 11 juvenile Aplysia elicited a brisk reflex withdrawal of the gill that readily habituated in response to repeated stimulation at a 10 sec ISI. Moreover, significant dishabituation was produced by a brief tail shock. These data were qualitatively similar to the habituation and dishabituation produced in the siphon withdrawal system of stage 11 animals with comparable-intensity tactile stimuli at the same $10 \mathrm{sec}$ ISI. One interesting difference, however, was that habituation in the gill withdrawal system was considerably more rapid, achieving an asymptotic habituation level of about $20 \%$ of initial response within 20 trials. A roughly comparable level of response decrement in the siphon system required more than 80 trials (Rankin and Carew, 1987). We do not yet understand this difference in habituation rates in the siphon and gill systems. Insights into this difference may be obtained by examining this question at the level of central neuronal correlates in the 2 response systems (see below).

\section{Central mediation of the gill withdrawal reflex}

An important consideration in producing preparations for a cellular analysis of learning is to determine the degree to which the reflex response to be studied is centrally mediated. To examine this question, we used a stimulus intensity to the siphon (4 mg) that we had previously established as capable of producing pronounced and significant habituation of the gill withdrawal reflex. By surgically removing the abdominal ganglia from intact animals and comparing them with sham-operated controls, we determined that the CNS accounted for almost all (about $95 \%$ ) of the gill withdrawal reflex in stage 11 juvenile animals.

These findings are in contrast to the earlier results of Lukowiak (1979), who reported that in young Aplysia (defined as 1.5-20 $\mathrm{gm}$ in weight) there was no change in the amplitude of the gill withdrawal reflex elicited by siphon stimulation in a semi-intact preparation when the abdominal ganglion was removed. The weight range in the study by Lukowiak would place the animals he examined into a considerably later stage of juvenile development (late stage 12; see Rankin et al., 1986) compared with the stage 11 animals in the present study. Nonetheless, there are clear differences in the estimated contribution of the CNS to the gill withdrawal reflex in the 2 studies.
From previous work it is known that a major factor influencing the contribution of the peripheral nervous system (PNS) to the gill withdrawal reflex in Aplysia is the stimulus intensity used to elicit the reflex: Stronger stimuli elicit a progrcssively greater peripheral contribution compared with weaker stimuli (Kupfermann et al., 1974; Peretz et al., 1976; Carew et al., 1979). In the study by Lukowiak (1979), a stimulus intensity of 1000 $\mathrm{mg}$ (delivered to the siphon via a mechanical tapper) was used to elicit the reflex. In our study, a stimulus intensity of $4 \mathrm{mg}$ was used. Thus, there is a difference in stimulus intensity in the 2 studies of more than 2 orders of magnitude. This difference is likely to contribute significantly to the different degrees of central contribution to the reflex that were observed in the 2 studies. However, although stimulus intensity appears to be the predominant factor, it is also possible that other factors, such as the type of stimuli used (i.e., mechanical tapper or water jet), may contribute to the observed differences (for a clear discussion of this general question, see Mpitsos and Lukowiak, 1986).

What is important for the present study, however, is that we have identified stimulus conditions in our experiments that permit examining a gill withdrawal reflex in juvenile animals that is mediated almost entirely (about 95\%) by the abdominal ganglion. Thus, using these stimuli, it is possible to analyze a response that one can confidently ascribe to the CNS.

\section{Relating behavioral and cellular responses in developing Aplysia}

To study the ontogeny of learning on a cellular level, it is important to have preparations in which it is possible to relate behavioral observations of learning directly to neuronal plasticity exhibited in the CNS. In the gill withdrawal system we have first shown that the intact animal exhibits 2 simple forms of learning: habituation in response to repeated tactile stimulation of the siphon and dishabituation in response to tail shock. We then developed a more simplified (semi-intact) behavioral preparation in which gill withdrawal could still be directly measured, but tactile stimulation of the siphon was replaced by weak electrical stimulation of the afferent nerve from the siphon (the siphon nerve) and tail shock was replaced by electrical stimulation of the central input pathway from the tail to the abdominal ganglion (the pleuroabdominal connectives). The habituation and dishabituation produced using nerve stimulation in the semiintact preparation were both qualitatively and quantitatively similar to those produced by physiological stimulation in the intact animal.

Finally, we developed an isolated CNS (abdominal ganglion) preparation in which subsequent detailed cellular and molecular studies would be possible. In this preparation the same nerve stimuli were used as in the semi-intact preparation (which in turn had been previously matched to behaviorally relevant stimuli in the intact animal). However, in the isolated CNS, as a substitute for recording gill withdrawal directly, we recorded the evoked neural discharge in an efferent nerve to the gill (the branchial nerve). Using this response measure, we again observed both qualitatively and quantitatively similar response decrement and facilitation in the isolated CNS as was seen behaviorally in the semi-intact preparation and the intact animal. Thus, our results indicate that, using the stimulation parameters we have identified, not only is the gill withdrawal reflex centrally mediated, but 2 simple forms of learning exhibited by this reflex can be localized to circuits in the abdominal ganglion. 


\section{Development of synaptic plasticity in the CNS}

A central question that remains to be established is whether it is possible to relate the emergence of different forms of learning during development to the emergence of different forms of synaptic plasticity. It has recently become clear in many systems that the emergence of plasticity in synaptic transmission represents a further step in neuronal differentiation, an elaboration of the basic mechanism of synaptic transmission itself. For example, Ohmori and his colleagues (Ohmori et al., 1981; Ohmori, 1982) showed that a fundamental form of synaptic plasticity, posttetanic potentiation, emerged at identified synapses in Aplysia long after synaptic transmission had been established at those synapses. In addition, Rayport and Kandel (1980) have studied the modulation of the efficacy of an identified electrical synapse between 2 giant neurons $\left(\mathrm{R}_{2}\right.$ and $\left.\mathrm{LP}_{1}\right)$ in Aplysia during juvenile development. They found that these neurons are strongly electrically coupled in stages 10 and 11 but become progressively more weakly coupled by late juvenile stage 12 and early stage 13 (adult). A similar finding in young crayfish has been reported by Fricke (1984), who described the weakening in coupling of the electrical synapses between the tactile sensory afferents and the lateral giant interneurons in the crayfish tail-flip circuit during juvenile development. This finding had the interesting functional implication that, as the escape reflex became progressively more controlled by chemical (rather than electrical) synaptic transmission, it became behaviorally more plastic, displaying habituation for the first time. Finally, Rayport and Camardo (1984) have shown that heterosynaptic facilitation of the synaptic input to the mucous motor neuron $\mathrm{R}_{2}$ in juvenile Aplysia emerges at a later developmental stage than synaptic transmission at this synapse. Moreover, they showed that plasticity at this synapse exhibits a clear developmental sequence: homosynaptic depression (produced by repeated activation of synaptic input) emerges at an earlier developmental stage (stage 9) than heterosynaptic facilitation, which appears about 4-7 d lat$\mathrm{er}$, in stage 10. Thus, there is considerable evidence to support the conclusion that plasticity at synapses can represent a later step in their differentiation. A fascinating question then is whether the emergence of synaptic plasticity in a particular neural circuit can account for the emergence of behavioral plasticity (learning) in the response systems mediated by that circuit.

Aplysia is an excellent system in which to address questions of the emergence of synaptic plasticity and its relationship to behavior. For example, the abdominal ganglion, which we have shown mediates the gill and siphon reflex of juveniles, is amenable to intracellular investigation at very early juvenile stages (Ohmori et al., 1981; Ohmori, 1982; Rayport and Camardo, 1984). In addition, a good deal is known about the development of the abdominal ganglion. Kriegstein (1977b) has described in detail the morphological changes that accompany its pre- and postmetamorphic development. He showed that significant anlagen of the abdominal ganglion, as well as the rest of the CNS, are present as early as the larval stage and described the migration and fusion of all the central ganglia as they assume their adult form. In addition to gill and siphon withdrawal, which is mediated by a neural circuit in the abdominal ganglion, Aplysia exhibits a variety of other behaviors that are mediated by other identified neural circuits. Moreover, many of these behaviors are known to exhibit different forms of learning. For example, tail withdrawal is mediatcd by circuits in the pleural and pedal ganglia (Walters et al., 1983; Cleary and Byrne, 1985); escape locomotion is mediated by circuits in the pedal and cerebral ganglia (Jahan-Parwar and Fredman, 1978a, b; Hening et al., 1979); and feeding is mediated by circuits in the cerebral and buccal ganglia (Kupfermann, 1974a, b). Thus, is should be possible in Aplysia to follow in detail the differentiation of identifiable regions of the CNS and correlate steps in the development of specific ganglia (and neural circuits within those ganglia) with the emergence of different behaviors and different forms of behavioral plasticity.

\section{Toward a cellular analysis of the development of learning} Nonassociative learning

An important question that can now be addressed concerns the time in development that the underlying mechanisms of dishabituation and sensitization first emerge. In the adult, dishabituation and sensitization are due to presynaptic facilitation, which enhances transmitter release from sensory neurons onto their follower cells (interneurons and motoneurons). An extensive body of work shows that the molecular mechanisms underlying sensitization involve cAMP-dependent protein phosphorylation, which results in closure of unique potassium channels (S-channels) in the sensory neurons that mediate this reflex. Closure of the $S$-channels causes broadening of the action potential, thereby increasing $\mathrm{Ca}^{2+}$ influx at the presynaptic terminals and the amount of transmitter released. Candidate facilitatory transmitters that activate this molecular cascade include serotonin (5-HT) and the small cardioactive peptides $\mathrm{SCP}_{\mathrm{A}}$ and $\mathrm{SCP}_{\mathrm{B}}$ (for reviews, see Kandel and Schwartz, 1982; Carew, 1986; Hawkins et al., 1986). In addition, the enhancement of synaptic transmission may also involve the increased mobilization of transmitter (Boyle et al., 1985; Gingrich and Byrne, 1985; Hochner et al., 1985). Thus, the emergence of presynaptic facilitation may involve the progressive assembly of various component parts of the above biophysical and molecular mechanisms, which in turn may be dissected and analyzed developmentally.

An obvious and fundamental qucstion is whether the emergence and maturation of presynaptic facilitation in the gill and siphon withdrawal circuit parallels the emergence of dishabituation and/or sensitization. Specifically, it will be important to determine the details of the biophysical and molecular events that are expressed in the withdrawal circuit at the same time that dishabituation and sensitization emerge behaviorally. There are a number of possibilities that can be tested. For example, it is possible that the $\mathrm{S}$-channels are absent or not functional in sensory neurons prior to stage 10 in juvenile development. Alternatively, the cyclic nucleotide-second messenger system may not be capable of activation by the facilitatory transmitterreceptor complex until stage 10 , or the relevant facilitating neurotransmitter systems (e.g., 5-HT or the peptides $\mathrm{SCP}_{\mathrm{A}}$ and $\mathrm{SCP}_{\mathrm{B}}$ ) may not yet be expressed. Consistent with this last possibility are the recent observations of Nolen et al. (1986), who used immunocytochemical techniques to localize 5-HT-containing neurons in the CNS of developing Aplysia. They found that there are very few 5-HT-immunoreactive neurons in the abdominal ganglion in stage 9 animals and that this number dramatically increases (triples) in stage 10 , when dishabituation in the siphon withdrawal reflex first emerges, suggesting the possibility that the development of specific serotonergic neurons in the abdominal ganglion may contribute to the emergence of this form of learning. Finally, it is also possible that there are 
novel mechanisms not yet identified in the adult which will be revealed by a developmental analysis.

\section{Associative learning}

In addition to nonassociative learning, the gill and siphon withdrawal reflex also exhibits different forms of associative learning such as classical conditioning (Carew et al., 1981, 1983), operant conditioning (Hawkins et al., 1985), and context conditioning (Colwill, 1985). The cellular mechanism of classical conditioning in this reflex involves activity-dependent presynaptic facilitation, which results in the enhancement of the same cellular mechanism, presynaptic facilitation, that underlies sensitization (Hawkins et al., 1983; see also Walters and Byrne, 1983). The mechanism underlying activity dependence is not yet known but is thought to involve $\mathrm{Ca}^{2+}$ entry into the neuron during activity, which in turn amplifies the cAMP cascade (Abrams et al., 1985; Ocorr et al., 1985). Based on these observations, it is important to address 2 questions. First, when classical conditioning emerges behaviorally during development, does activity dependence in the neural circuit for gill and siphon withdrawal also emerge in parallel? Second, when activity dependence emerges, what specific biophysical or molecular processes are added to those underlying sensitization that might account for the emergence of classical conditioning? It is possible, for example, that $\mathrm{Ca}^{2+} /$ calmodulin dependence for the enhancement of adenylate cyclase (Abrams et al., 1985) is first expressed when activity dependence emerges or that the $\mathrm{Ca}^{2+}$ component of the action potential is enhanced at that time in development. Clearly these ideas are speculative. They arc described primarily to illustrate that our understanding of the cellular and molecular mechanisms of learning in adult Aplysia suggest several clear and testable hypotheses concerning mechanisms underlying the ontogeny of learning during development.

It is important to emphasize that we are just at the beginning of this experimental approach, and a number of problems remain to be addressed before we can explore the mechanistic questions we have described. Most importantly, in order to carry out these cellular studies it is necessary to identify the sensory and motor neurons, as well as interneurons, that make up the reflex circuit in juvenile animals. Fortunately, the reflex is intact as soon as the siphon and gill emerge in their respective developmental stages (Rankin et al., 1987), indicating that at least some aspects of the neural circuit are functional at these specifiable developmental stages. Moreover, even in the earliest juvenile stages, the abdominal ganglion, as well as the rest of the CNS, is quite amenable to a variety of neuroanatomical and immunocytochemical approaches (Kriegstein, 1977a, b; Goldstein et al., 1984; Nolen et al., 1986) that will permit identification of sensory and motor neurons, as well as facilitating interneurons in the neural circuit underlying the withdrawal reflex. Thus, we are encouraged by the tractability of juvenile Aplysia for developmental studies and are optimistic that development in Aplysia can serve as a useful tool with which to isolate and analyze the components of various forms of learning and memory as they are assembled during ontogeny.

\section{References}

Abrams, T. W., L. Eliot, Y. Dudai, and E. R. Kandel (1985) Activation of adenylate cyclase in Aplysia neural tissue by $\mathrm{Ca}^{2+} /$ calmodulin, a candidate for an associative mechanism during conditioning. Soc. Neurosci. Abstr. 11: 797.

Amsel, A., and M. Stanton (1980) Ontogeny and phylogeny of para- doxical reward effects. In Advances in the Study of Behavior, Vol. 2, pp. 227-274, Academic, New York.

Boyle, M. B., M. Klein, S. J. Smith, and E. R. Kandel (1985) Serotonin increases intracellular $\mathrm{Ca}^{2+}$ transient in voltage-clamped sensory neurons in Aplysia californica. Proc. Natl. Acad. Sci. USA 81: 76427646.

Campbell, B. A., and X. Coulter (1976) The ontogenesis of learning and memory. In Neural Mechanisms of Learning and Memory, M. R. Rosenzweig and E. L. Bennett, eds., pp. 71-74, MIT Press, Cambridgc, MA.

Campbell, B. A., and N. E. Spear (1972) Ontogeny of memory. Psychol. Rev. 79(3): 215-236.

Carew, T. J. (1986) Advances in the cellular analysis of learning in Aplysia. In Cellular Basis of Learning: Dahlem Konferenzen, J.-P. Changeux and M. Konishi, eds., Springer (in press).

Carew, T. J., V. Castellucci, J. H. Byrne, and E. R. Kandel (1979) A quantitative analysis of the relative contribution of central and peripheral neurons to the gill-withdrawal reflex in Aplysia. J. Neurophysiol. 42: 497-509.

Carew, T. J., E. T. Walters, and E. R. Kandel (1981) Classical conditioning in a simple withdrawal reflex in Aplysia. J. Neurosci. 1: 1426-1437.

Carew, T. J., R. D. Hawkins, and E. R. Kandel (1983) Differential classical conditioning of a defensive withdrawal reflex in Aplysia californica. Science 219: 397-400.

Cleary, L. J., and J. H. Byrne (1985) Interneurons contributing to the mediation and modulation of the tail-withdrawal reflex in Aplysia. Soc. Neurosci. Abstr. 11: 642.

Colwill, R. M. (1985) Context conditioning in Aplysia californica. Soc. Neurosci. Abstr. 11: 796.

Fricke, R. A. (1984) Development of habituation in the crayfish due to selective weakening of electrical synapses. Brain Res. 322: 139143.

Gingrich, K. J., and J. H. Byrne (1985) Simulation of synaptic depression, posttetanic potentiation, and presynaptic facilitation of synaptic potentials from sensory neurons mediating gill-withdrawal reflex in Aplysia. J. Neurophysiol. 53: 652-669.

Goldstein, R., H. B. Kistler, Jr., H. Steinbush, and J. H. Schwartz (1984) Distribution of serotonin-immunoreactivity in juvenile Aplysia. Neuroscience 11: 535-547.

Hawkins, R. D., T. W. Abrams, T. J. Carew, and E. R. Kandel (1983) A cellular mechanism of classical conditioning in Aplysia activitydependent amplification of presynaptic facilitation. Science 219:400405.

Hawkins, R. D., G. A. Clark, and E. R. Kandel (1985) Operant conditioning and differential classical conditioning of gill withdrawal in Aplysia. Soc. Neurosci. Abstr. 11: 796.

Hawkins, R. D., G. A. Clark, and E. R. Kandel (1986) Cell biological studies of learning in simple vertebrate and invertebrate systems. In Handbook of Physiology, Section I. The Nervous System, Vol. 6: Higher Functions of the Nervous System, F. Plum, ed., American Physiological Society, Bethesda, MD (in press).

Hening, W. A., E. T. Walters, T. J. Carew, and E. R. Kandel (1979) Motor neuronal control of locomotion in Aplysia. Brain Res. 179: 231-253.

Hochner, B., S. Schacher, M. Klein, and E. R. Kandel (1985) Presynaptic facilitation in Aplysia sensory neurons: A process independent of $\mathrm{K}^{+}$current modulation becomes important when transmitter release is depressed. Soc. Neurosci. Abstr. 11: 29.

Jahan-Parwar, B., and S. M. Fredman (1978a) Pedal locomotion in Aplysia. I. Sensory and motor function of foot nerves. Comp. Biochem. Physiol. [A] 60: 459-465.

Jahan-Parwar, B., and S. M. Fredman (1978b) Pedal locomotion in Aplysia. II. Proprioceptive and tactile reflexes. J. Neurophysiol, 41: $600-608$.

Kandel, E. R., and J. H. Schwartz (1982) Molecular biology of learning: Modulation of transmitter release. Science 218: 433-442.

Kriegstein, A. R. (1977a) Stages in the post-hatching development of Aplysia californica. J. Exp. Zool. 199: 275-288.

Kriegstein, A. R. (1977b) Development of the nervous system of Aplysia californica. Proc. Natl. Acad. Sci. USA 74: 375-378.

Kriegstein, A. R., V. Castellucci, and E. R. Kandel (1974) Metamorphosis of Aplysia californica in laboratory culture. Proc. Natl. Acad. Sci. USA 71: 3654-3658.

Kupfermann, I. (1974a) Feeding behavior in Aplysia: A simple system 
for the study of motivation. Behav. Biol. 10:1-26.

Kupfermann, I. (1974b) Dissociation of the appetitive and consummatory phases of feeding behavior in Aplysia. A lesion study. Behav. Biol. 10: 89-97.

Kupfermann, I., T. J. Carew, and E. R. Kandel (1974) Local, reflexive and central commands controlling gill and siphon movements in Aplysia californica. J. Neurophysiol. 37: 966-1010.

Lukowiak, K. (1979) The development of central nervous system control of the gill withdrawal reflex evoked by siphon stimulation in Aplysia. Can. J. Physiol. Pharmacol. 57: 987-997.

Mpitsos, G. J., and K. Lukowiak (1986) Learning in gastropod molluscs. In The Mollusca, Vol. 8: Neurobiology and Behavior, Part 1, A. O. D. Willows, ed., pp. 96-267, Academic, Orlando, FL.

Nolen, T. G., E. A. Marcus, and T. J. Carew (1985) Development of learning and memory in Aplysia. III: Central neuronal correlates. Soc. Neurosci. Abstr. 11: 643.

Nolen, T. G., J. A. Mindell, and T. J. Carew (1986) Development of neurotransmitters implicated in learning in Aplysia. Soc. Neurosci. Abstr. 12: 399.

Ocorr, K. A., E. T. Walters, and J. H. Byrne (1985) Associative conditioning analog selectively increases cAMP levels of tail sensory neurons in Aplysia. Proc. Natl. Acad. Sci. USA 82: 2538-2552.

Ohmori, H. (1982) Development of post-tetanic potentiation at identified inhibitory and excitatory synapses in Aplysia. J. Physiol. (Lond.) 322: 223.

Ohmori, H., S. G. Rayport, and E. R. Kandel (1981) Emergence of post-tetanic potentiation as a distinct phase in the differentiation of an identified synapse in Aplysia. Science 213: 1016.

Peretz, B., J. W. Jacklet, and K. Lukowiak (1976) Habituation of reflexes in Aplysia, contribution of the peripheral and central nervous systems. Science 191: 396-399.

Rankin, C. H., and T. J. Carew (1987) Development of learning and memory in Aplysia. II. Habituation and dishabituation. J. Neurosci. 7: 133-143.

Rankin, C. H., M. Stopfer, E. A. Marcus, and T. J. Carew (1987) Development of leaming and memory in Aplysia. I. Functional assembly of gill and siphon withdrawal. J. Neurosci. 7: 120-132.

Rayport, S., and J. S. Camardo (1984) Differential emergence of cellular mechanisms mediating habituation and sensitization in the developing Aplysia nervous system. J. Neurosci. 4: 2528-2532.

Rayport, S., and E. R. Kandel (1980) Developmental modulation of an identified electrical synapse: Functional uncoupling. J. Neurophysiol. 44: 555-567.

Walters, E. T., and J. H. Byrne (1983) Associative conditioning of a single sensory neuron suggests a cellular mechanism for learning. Science 219: 405-408.

Walters, E. T., J. H. Byrne, T. J. Carew, and E. R. Kandel (1983) Mechanoafferent neurons innervating the tail of Aplysia. I. Response properties and synaptic connections. J. Neurophysiol. 50:1522-1542. Winer, B. (1962) Statistical Principles in Experimental Design, McGraw-Hill, New York. 